\title{
Relações entre o Pensamento Computacional e a Matemática: uma Revisão Sistemática da Literatura
}

\author{
Thiago Barcelos ${ }^{1,4}$, Roberto Muñoz $^{2,3}$, Rodolfo Villarroel ${ }^{3}$, Ismar Silveira \\ ${ }^{1}$ Instituto Federal de Educação, Ciência e Tecnologia de São Paulo - IFSP \\ Av. Salgado Filho, 3501, Guarulhos, SP, Brasil - CEP 07115-000 \\ ${ }^{2}$ Universidad de Valparaíso - Escuela de Ingeniería Civil Informática \\ General Cruz, 222, Valparaíso, Chile - CP 2362905 \\ ${ }^{3}$ Pontificia Universidad Católica de Valparaíso - PUCV \\ Brasil, 2241, Valparaíso, Chile - CP 2362807 \\ ${ }^{4}$ Universidade Presbiteriana Mackenzie \\ Rua da Consolação, 930 - São Paulo, SP, Brasil - CEP 01302-907 \\ tsbarcelos@ifsp.edu.br; roberto.munoz.s@uv.cl ; \\ rodolfo.villarroelducv.cl; ismar@mackenzie.br
}

\begin{abstract}
A better understanding of the relationships between Computational Thinking and disciplines already present in the school curriculum may help the identification of possible educational benefits. In this sense, this article presents a Systematic Literature Review (SLR) that includes studies published between 2006 and 2014. The 48 included studies describe and evaluate didactic activities that develop Computational Thinking together with skills or contents related to Math. A wide variety of mathematical topics is being developed, with some emphasis on Algebra, Calculus and also higher-order thinking skills. In the last two years there was an increase in the number of activities focused on basic educational levels. Also, more rigorous methodological procedures have been used to evaluate learning effects. On the other hand, there are few studies focused on Math modelling and teacher training.
\end{abstract}

Resumo. Uma melhor compreensão das relações entre o Pensamento Computacional e disciplinas já presentes no currículo da educação básica pode contribuir para a identificação de possíveis benefícios educacionais. Dessa forma, este artigo apresenta uma Revisão Sistemática da Literatura (RSL), incluindo 48 estudos publicados em língua inglesa entre 2006 e 2014 que apresentam atividades didáticas desenvolvendo o Pensamento Computacional e competências, habilidades ou conteúdos da Matemática. Vários tópicos matemáticos são desenvolvidos, com predominância da Álgebra, Cálculo e habilidades cognitivas de alto nível. Verifica-se ainda, nos últimos dois anos, um aumento do desenvolvimento de experiências na educação básica e um maior rigor metodológico na avaliação dos efeitos de aprendizagem. Por outro lado, há uma carência de estudos relacionados à Modelagem Matemática e à formação de professores. 
CBIE-LACLO 2015

Anais dos Workshops do IV Congresso Brasileiro de Informática na Educação (CBIE 2015)

\section{Introdução}

A comunidade de pesquisa em Informática na Educação tem considerado que a Ciência da Computação deveria fazer parte do currículo escolar desde as séries iniciais, sendo assim posicionada no mesmo nível das chamadas "ciências básicas", como a Física, a Biologia e a Química. No entanto, a motivação para essa proposta tem sido motivada pela Ciência da Computação em si, já que o principal incentivo para ensinar habilidades relacionadas à Computação tem sido promover a fluência digital entre os estudantes. Hood e Hood afirmam que a chave para obter a fluência dos estudantes em Tecnologia da Informação é incorporá-la ao currículo escolar (HOOD; HOOD, 2005). Porém tal fluência não deveria ocorrer por intermédio do domínio de um conjunto de técnicas, mas sim por uma organização do pensamento voltada à resolução de problemas.

Por essa razão, um conjunto de competências e habilidades associadas à Ciência da Computação deveria ser desenvolvido pelos estudantes desde os primeiros anos da educação básica. Wing nomeou tal conjunto como Pensamento Computacional (WING, 2006). Esse termo é atualmente utilizado para descrever os processos cognitivos relacionados à abstração e decomposição para permitir a resolução de problemas utilizando recursos computacionais e estratégias algorítmicas, dentre outras habilidades. A definição foi criticada por alguns autores por ser muito abrangente (HU, 2011; HEMMENDINGER, 2010). No entanto, é possível identificar imediatamente algumas convergências entre essa definição e competências e habilidades relacionadas à Matemática. Polya, em seu clássico trabalho sobre a resolução de problemas matemáticos, apontou que a abstração (definida como a combinação de analogia, generalização e especialização) e a decomposição de problemas são cruciais para o sucesso na resolução de problemas (POLYA, 2004).

Por outro lado, a Matemática se constitui como um obstáculo para os estudantes de vários países, em particular os latino-americanos. Conforme Aedo e Walker (2012), a pontuação média dos exames de Matemática do PISA na Argentina, Brasil, Chile, México e Perú é aproximadamente 100 pontos inferior à pontuação média obtida nos outros países que compõem a OECD (Organization for Economic Co-operation Development). Isso equivale a uma defasagem de cerca de dois anos na educação dos estudantes dos cinco países citados anteriormente.

Para incorporar atividades de desenvolvimento do Pensamento Computacional na educação básica é necessário considerar qual seria o impacto de tais atividades na aprendizagem dos conteúdos escolares tradicionais. Isso é particularmente importante por ser o ensino básico, em vários países, um ambiente no qual várias prioridades, ideologias e filosofias lutam por atenção (CSTA, 2011). Relações entre a Matemática e a Ciência da Computação no ambiente educacional já foram discutidas anteriormente (KE, 2014; KRONE; SITARAMAN; HALLSTROM, 2011). Dessa forma, é razoável inferir que o processo de ensino-aprendizagem de Matemática seria beneficiado de alguma forma pela incorporação do Pensamento Computacional ao currículo do ensino básico.

Muitas atividades visando o desenvolvimento do Pensamento Computacional associado a competências, habilidades e conteúdos matemáticos foram reportadas na literatura nos últimos anos, o que motiva uma análise detalhada dos resultados educacionais obtidos por intermédio dessas iniciativas. Dessa forma, este artigo apresenta uma revisão sistemática da literatura com o objetivo de identificar como as 
relações entre a Matemática e o Pensamento Computacional foram demonstradas por meio do desenvolvimento de atividades didáticas descritas na literatura. A revisão foi guiada pelas seguintes perguntas de pesquisa:

(Q1) Como atividades didáticas relacionadas ao Pensamento Computacional e à Matemática foram desenvolvidas e qual seu público-alvo?

(Q2) Que competências, habilidades e conteúdos do Pensamento Computacional e da Matemática são ensinados nas atividades didáticas?

(Q3) Que métodos e técnicas de pesquisa são empregados para identificar possíveis efeitos de aprendizagem?

\section{Métodos}

Para o desenvolvimento da revisão sistemática seguimos as diretrizes sistematizadas por Wohlin et al. (2012), enumeradas a seguir:

Estágio 1. Planejamento da revisão, envolvendo (1.1) Identificação da necessidade de uma revisão; (1.2) Definição da pergunta de pesquisa e (1.3) Definição do protocolo da revisão.

Estágio 2. Condução da revisão, envolvendo (2.1) Identificação das pesquisas, (2.2) Seleção de estudos primários, (2.3) Verificação da qualidade dos estudos, (2.4). Extração e monitoramento de dados e (2.5) Síntese de dados.

Estágio 3. Relato dos resultados.

\subsection{Planejamento da revisão}

Inicialmente, uma pesquisa preliminar foi realizada em três repositórios online (ACM, IEEE e Google Acadêmico), a partir da qual não foi encontrada uma revisão da literatura com o mesmo objetivo da proposta deste trabalho. Assim, esta pesquisa foi útil para indicar a necessidade de uma revisão (Atividade 1.1). As questões de pesquisa para a revisão (Atividade 1.2) e as suas motivações já foram descritas na seção 1 do artig.

O passo seguinte está relacionado com a definição do protocolo da revisão (Atividade 1.3). Segundo Wohlin et al. (2012) o protocolo deve indicar a estratégia de pesquisa, que inclui os repositórios de pesquisa dos quais serão extraídos os estudos, definição de strings de busca e critérios de inclusão e exclusão. Cinco repositórios foram incluídos nesta revisão: ACM, IEEE Xplore, ERIC, ScienceDirect e SpringerLink. A pesquisa sobre as características e atividades do Pensamento Computacional é razoavelmente recente e, por isso, optamos por usar uma string de busca abrangente: "computational thinking" and ("math" or "mathematics"). O objetivo foi identificar a maior quantidade de estudos possível, disponíveis em língua inglesa, mesmo à custa de uma seleção manual mais trabalhosa.

Foram definidos dois critérios de inclusão. Para ser incluído na revisão, o estudo deveria (CI1) indicar alguma relação entre o Pensamento Computacional e alguma competência, habilidade ou tópico relacionado à Matemática. Ainda, para ser incluído na revisão um estudo deveria $(\mathrm{CI} 2)$ descrever uma atividade didática e apresentar os resultados da sua avaliação. Como optamos por definir uma estratégia de busca inclusiva, foram incluídos tanto estudos que apresentaram resultados de experimentos 
CBIE-LACLO 2015

Anais dos Workshops do IV Congresso Brasileiro de Informática na Educação (CBIE 2015)

controlados quanto estudos que apresentavam apenas evidências empíricas. Essa estratégia foi adotada para identificar quão precisa foi a identificação dos resultados educacionais. Da mesma forma, foram excluídos estudos que atendiam a algum dos critérios de exclusão abaixo:

(CE1) O artigo descreve um tutorial, demonstração, proposta de painel de discussão ou entrevista;

(CE2) Pensamento Computacional (ou o ensino de Computação) é o tema do artigo, no entanto não são apresentadas relações com a Matemática;

(CE3) O artigo está fora de contexto e aborda outro tema de pesquisa.

\subsection{Condução da revisão}

As buscas foram executadas entre 11 e 18 de abril de 2014 nos cinco repositórios selecionados, quando 771 documentos foram obtidos. Os primeiros dois autores deste artigo foram responsáveis por classificar os artigos baseando-se no título e resumo. Quando após este procedimento ainda não era claro se o artigo poderia ser incluído na revisão, ele era marcado para posterior revisão do seu texto completo. Ou seja, após a primeira fase da análise, os artigos eram classificados como incluído (I), excluído (E) ou marcado para verificação (V). Na segunda fase, o texto completo dos artigos do último grupo foi analisado, e a classificação final de tais artigos foi: incluído após verificação (IV) ou excluído após verificação (EV). As estatísticas de inclusão e exclusão após as duas fases da análise são apresentadas na Tabela 1.

Tabela 1. Estatísticas dos artigos obtidos.

\begin{tabular}{|c|c|c|c|c|c|}
\hline \multirow[b]{2}{*}{ Database } & \multicolumn{2}{|c|}{ Artigos incluídos } & \multicolumn{2}{|c|}{ Artigos excluídos } & \multirow{2}{*}{ Total } \\
\hline & $\mathbf{I}$ & IV & $\mathbf{E}$ & EV & \\
\hline $\mathrm{ACM}$ & 28 & 5 & 381 & 22 & 436 \\
\hline ERIC & 3 & 0 & 8 & 0 & 11 \\
\hline IEEE Xplore & 10 & 10 & 106 & 16 & 142 \\
\hline ScienceDirect & 5 & 3 & 17 & 9 & 34 \\
\hline SpringerLink & 11 & 2 & 127 & 8 & 148 \\
\hline Total & \multicolumn{2}{|c|}{77} & \multicolumn{2}{|c|}{694} & 771 \\
\hline
\end{tabular}

Pode-se verificar que a maioria dos artigos obtidos foi excluída da revisão - 694 de 771 documentos, aproximadamente $90 \%$ do total. A maioria das exclusões (369) ocorreu por artigos nos quais os termos da busca eram citados mas nenhuma relação entre os conceitos era discutida (critério CE3). 242 artigos foram excluídos por estarem fora de contexto (EC3) e 82 artigos por apresentarem apenas o resumo de uma discussão ou sessão presencial (EC1).

Os 77 artigos incluídos foram então separados em dois grupos: o primeiro grupo (EXP), com 48 artigos, incluiu os estudos que descreveram a aplicação de uma atividade didática acompanhada de uma avaliação experimental dos resultados de aprendizagem. O segundo grupo (DI) foi formado por 29 artigos nos quais os autores apresentavam discussões conceituais das relações entre o Pensamento Computacional e a Matemática com base em seu ponto de vista, análise documental ou outro tipo de 
estudo teórico. Também foram incluídos neste grupo os artigos que descreviam propostas de atividades didáticas sem nenhuma descrição de sua aplicação ou avaliação. Por limitação de espaço, a partir deste ponto serão apresentadas apenas as análises realizadas nos 48 artigos do primeiro grupo.

\subsection{Relato dos resultados}

Em cada artigo do grupo EXP foi identificado qual o público-alvo da atividade ou proposta relatada. Foi adotada na classificação a terminologia norte-americana para os níveis de ensino: elementary school, para os cinco primeiros anos da educação básica; middle school, para os três anos subsequentes da educação básica; e high school, para os quatro anos finais. Em geral, os autores de artigos que realizam experiências fora do contexto norte-americano também utilizaram essa terminologia para reportar suas experiências. Para os artigos em que essa estratégia não foi utilizada, o nível de ensino foi deduzido a partir da leitura de modo a uniformizar a contagem. Os dados são apresentados na Tabela 2 .

Tabela 2. Segmentação de estudos por nível de ensino do público-alvo.

\begin{tabular}{|l|c|c|}
\hline Nível & Quantidade & Percentual (n=48) \\
\hline Single audience: & 5 & $10.42 \%$ \\
\hline Elementary School & 11 & $22.92 \%$ \\
\hline Middle School & 6 & $12.50 \%$ \\
\hline High School & 16 & $33.33 \%$ \\
\hline Graduação & 3 & $6.25 \%$ \\
\hline Professores & \multicolumn{2}{|c|}{} \\
\hline Públicos mistos: & 2 & $4.17 \%$ \\
\hline Elementary School / Middle School & 2 & $4.17 \%$ \\
\hline Middle School / High School & 1 & $2.08 \%$ \\
\hline High School / Graduação & 1 & $2.08 \%$ \\
\hline Graduação / Professores & 1 & $2.08 \%$ \\
\hline Graduação / Middle School & 48 & $100.00 \%$ \\
\hline Total & & \\
\hline
\end{tabular}

É possível identificar que uma expressiva quantidade de estudos (26 artigos) apresentam experiências voltadas a um ou mais níveis da educação básica. Por outro lado, foram identificados 16 artigos que tem como alvo exclusivamente o ensino de graduação, ou tiveram sua validação experimental realizada neste ambiente. Apesar da definição original de Pensamento Computacional (WING, 2006) considerar o seu desenvolvimento como uma proposta voltada à educação básica, muitos pesquisadores vem também propondo atividades voltadas ao desenvolvimento do Pensamento Computacional de forma aliada com a Matemática para alunos de graduação. Tais atividades são predominantemente voltadas para disciplinas introdutórias, visando atenuar os altos índices de evasão e reprovação em tais disciplinas. No entanto, também é necessário ressaltar que o interesse da comunidade em realizar experimentos na 


\section{CBIE-LACLO 2015}

Anais dos Workshops do IV Congresso Brasileiro de Informática na Educação (CBIE 2015)

educação básica vem crescendo: dos 26 artigos que relatam tais experiências, 10 (38.4\% do total) foram publicados apenas entre os anos de 2013 e 2014.

As experiências didáticas que desenvolvem a Matemática juntamente com o Pensamento Computacional vêm utilizando, predominantemente, ferramentas computacionais durante o seu desenvolvimento. A partir do mapeamento das ferramentas e materiais utilizados nos artigos, foram identificadas 42 ocorrências do uso de ferramentas computacionais e apenas 10 ocorrências do uso de atividades que não dependem do computador. Na Tabela 3 são apresentados as ferramentas de software utilizadas e os materiais físicos utilizados, bem como as respectivas frequências de utilização. É preciso ressaltar que um estudo pode apresentar mais de uma ocorrência de uso de ferramentas de software e materiais.

Tabela 3. Ferramentas de software e materiais utilizados em atividades didáticas.

\begin{tabular}{|l|c|}
\hline Software & Ocorrências \\
\hline Scratch & 11 \\
\hline $\begin{array}{l}\text { Linguagens de programação (Phyton, Java, } \\
\text { MATLAB, Octave, Lisp) }\end{array}$ & 6 \\
\hline $\begin{array}{l}\text { Objetos de aprendizagem de finalidade } \\
\text { específica }\end{array}$ & 4 \\
\hline Planilha eletrônica & 5 \\
\hline NetLogo / Logo & 6 \\
\hline Kit de Robótica, eletrônica ou controle físico & 1 \\
\hline AgentCubes / AgentSheets & 4 \\
\hline Ferramenta não mencionada & $\mathbf{4 2}$ \\
\hline TOTAL & $\mathbf{1 0}$ \\
\hline Material & Ocorrências \\
\hline Atividades CS Unplugged & 3 \\
\hline Atividades Lápis-e-papel & 7 \\
\hline TOTAL & 6 \\
\hline
\end{tabular}

Um variado conjunto de ferramentas computacionais é utilizado para o desenvolvimento de atividades. Das 42 ocorrências identificadas, pelo menos 26 referem-se a linguagens (ex: Java, MATLAB, Phyton), ambientes (ex: Scratch, AgentSheets) ou aplicações que permitem que o aluno desenvolva seus próprios artefatos computacionais. As atividades de "computação desplugada", que utilizam o conjunto de materiais CS Unplugged (BELL et al., 2010), são utilizadas na formação de professores (JENKINS; JERKINS; STENGER, 2012) e como um estudo de caso para avaliar a opinião de alunos sobre a associação entre a Matemática e a Ciência da Computação (TAUB; ARMONI; BEN-ARI, 2012).

A seguir foram analisadas as habilidades e conteúdos relacionados à Matemática que vem sendo desenvolvidos em conjunto com o Pensamento Computacional nos estudos realizados. A partir do agrupamento dos estudos que desenvolvem temas 
semelhantes foi possível identificar oito grupos de habilidades e conteúdos desenvolvidos:

- Habilidades cognitivas de alto nível: 14 estudos;

- Álgebra e Cálculo: 18 estudos;

- Álgebra Linear: 11 estudos

- Geometria Planar: 11 estudos;

- Aritmética: 9 estudos;

- Física: 7 estudos;

- Estatística: 6 estudos;

- Modelagem Matemática: 3 estudos;

Novamente deve-se observar que um mesmo estudo pode abordar habilidades ou conteúdos em mais de um grupo (por exemplo, Álgebra e Física). O primeiro grupo de estudos mencionado enfatiza o desenvolvimento de habilidades cognitivas mais gerais, discutindo suas relações com a Matemática. Dentre as habilidades desenvolvidas encontram-se a abstração e generalização para resolução de problemas (JENKINS; JERKINS; STENGER, 2012), identificação de padrões (BOYCE et al., 2011) e a colaboração entre equipes por intermédio da linguagem matemática (MAXWELL et al., 2013). Uma ampla variedade de conteúdos matemáticos vem sendo abordada, com alguma predominância para atividades que desenvolvem tópicos relacionados à Álgebra e ao Cálculo. Isso pode ser associado ao frequente uso de linguagens de programação e ambientes que induzem o uso de uma sintaxe baseada em conceitos algébricos como as variáveis. Além disso, de forma até esperada, os estudos que abordam o desenvolvimento de tópicos de Aritmética e Geometria Planar são predominantemente voltados ao público alvo da educação fundamental (elementary school), enquanto que os estudos que abordam tópicos de Álgebra e Cálculo, Estatística e Álgebra Linear são predominantemente voltados para alunos do ensino médio (high school) e de graduação.

Por fim, os estudos foram analisados em relação ao rigor dos métodos e técnicas de pesquisa empregados na avaliação da aprendizagem. Dessa forma, os estudos foram separados em dois grupos: o primeiro contém os estudos que apresentam uma avaliação da aprendizagem formal, sob o ponto de vista do rigor da metodologia empregada. No caso de estudos que adotam o paradigma quantitativo, foram considerados como formais aqueles estudos que empregam um experimento, com atribuição aleatória de indivíduos em grupos de controle e experimental, ou quase-experimento, com a comparação entre grupos não-equivalentes, sem atribuição aleatória dos indivíduos a cada grupo (TROCHIM; DONELLY, 2006). Para avaliar o rigor dos estudos que adotam o paradigma qualitativo utilizamos dois dos critérios de validação propostos por Creswell e Miller (2000), denominados "engajamento prolongado em campo", entendido como o envolvimento do pesquisador no ambiente pesquisado por um período entre quatro meses e um ano, e a "descrição densa e rica" dos fenômenos observados, incluindo o ambiente e as falas dos sujeitos pesquisados. A avaliação foi feita de forma independente por dois dos autores deste artigo e eventuais discrepâncias na avaliação foram identificadas de forma conjunta a posteriori. Os resultados são apresentados na Tabela 4.

Tabela 4. Nível de rigor na avaliação dos efeitos de aprendizagem. 
CBIE-LACLO 2015

Anais dos Workshops do IV Congresso Brasileiro de Informática na Educação (CBIE 2015)

\begin{tabular}{|l|c|c|}
\hline $\begin{array}{l}\text { Formal } \\
\text { (experimento, quase-experimento ou estudo qualitativo rigoroso) }\end{array}$ & 33 & $68.75 \%$ \\
\hline $\begin{array}{l}\text { Informal } \\
\text { (estudo empírico ou observacional, ou amostra de tamanho insuficiente) }\end{array}$ & 15 & $31.25 \%$ \\
\hline Total & 48 & $100.00 \%$ \\
\hline
\end{tabular}

É possível identificar uma crescente tendência no uso de uma avaliação mais rigorosa dos efeitos de aprendizagem obtidos a partir de atividades didáticas envolvendo o CT. Dos 33 artigos que apresentam avaliação formal, um terço deles (11 artigos) foi publicado somente nos anos de 2013 e 2014, enquanto que no mesmo período foram identificados apenas 3 artigos com validação informal.

\section{Discussão}

A partir da análise dos estudos incluídos nesta revisão é possível inferir que há um crescente interesse da comunidade científica em explorar as relações entre $\mathrm{o}$ Pensamento Computacional e a Matemática. O aumento significativo, nos últimos anos, na quantidade de estudos nos quais atividades didáticas são apresentadas e testadas empiricamente contribui para essa conclusão.

Apesar de, originalmente, o conceito de Pensamento Computacional referir-se a habilidades que deveriam ser desenvolvidas por estudantes da educação básica, verificou-se que as atividades didáticas descritas não se restringem apenas esse nível de ensino: cerca de $33 \%$ das atividades didáticas relatadas foram oferecidas a estudantes de graduação. Por um lado, isso indica que o desenvolvimento do Pensamento Computacional tem se mostrado útil na organização das pesquisas que tem como finalidade resolver os problemas de evasão e reprovação em cursos de graduação em Computação. Entretanto, isso não deixa de ser um "desvio" nos objetivos originais do Pensamento Computacional, já que o ensino de graduação é tipicamente o campo de aplicação mais próximo da realidade da pesquisa nas universidades. Algumas experiências didáticas apresentadas nos estudos têm como objetivo a formação de professores para disseminar o desenvolvimento do Pensamento Computacional; no entanto, tais iniciativas ainda são muito preliminares (apenas 4 estudos em um universo de 48) e necessitam de uma maior sistematização.

Verifica-se um aumento do interesse da comunidade pela condução de experimentos didáticos na educação básica nos últimos dois anos (2013 e 2014) abrangidos pela revisão. Da mesma forma, o rigor metodológico empregado na avaliação da aprendizagem também parece ter aumentado nos estudos publicados nos últimos dois anos. As atividades didáticas desenvolvidas se relacionam a uma grande variedade de conteúdos matemáticos, utilizando ferramentas computacionais também bastante diversas. Esse é um indicativo da flexibilidade e potencial dos conceitos e ferramentais computacionais como suporte para ensinar e contextualizar a Matemática. Essa evidência contradiz parcialmente a revisão da literatura apresentada por (GROVER; PEA, 2013), que menciona que o Pensamento Computacional não vinha sendo utilizado para ensinar outras disciplinas. Entretanto, deve-se notar a carência de trabalhos que utilizam a estratégia da Modelagem Matemática, visto que a construção e interpretação de modelos podem ser consideradas como uma habilidade comum à Matemática e ao Pensamento Computacional (BARCELOS; SILVEIRA, 2012; LEE et al., 2011). 
CBIE-LACLO 2015

Anais dos Workshops do IV Congresso Brasileiro de Informática na Educação (CBIE 2015)

\section{Conclusões}

O Pensamento Computacional representa um conjunto de habilidades relacionadas à Ciência da Computação que deveriam ser desenvolvidas pelos estudantes da educação básica. No entanto, se mostra necessário compreender as relações do Pensamento Computacional com as disciplinas tradicionais do currículo escolar e quais os possíveis benefícios do desenvolvimento de estratégias didáticas conjuntas. Neste artigo apresentamos uma revisão sistemática da literatura de estudos publicados entre 2006 e 2014 que abordam as relações entre o Pensamento Computacional e a Matemática e cujos resultados permitem identificar os avanços e limitações da pesquisa nessa área.

A maioria das experiências didáticas descritas tem como público-alvo os alunos da educação básica, mas uma parcela relevante das experiências foi desenvolvida com alunos de graduação. Por outro lado, há relatos insuficientes de experiências desenvolvidas para formação inicial e continuada de professores. Uma grande variedade de tópicos da Matemática vem sendo abordados, com alguma predominância para a Álgebra e o Cálculo. Vários estudos procuram desenvolver conjuntamente o Pensamento Computacional a Matemática por meio de habilidades de alto nível compartilhadas entre os dois paradigmas de pensamento, mas poucos estudos utilizam a construção e avaliação de modelos matemáticos e computacionais.

É possível identificar um claro avanço na disponibilidade e variedade de atividades didáticas que envolvem o Pensamento Computacional e a Matemática. No entanto, ainda há públicos-alvo e habilidades matemáticas que vem sendo pouco exploradas pela comunidade. Por meio da identificação dessas limitações, esperamos que novos estudos venham a suprir essas lacunas. Em trabalhos futuros, pretende-se desenvolver uma análise mais aprofundada das técnicas de pesquisa empregadas nos estudos incluídos na revisão, bem como estendê-la incluindo também estudos publicados em português e espanhol.

\section{Agradecimentos}

Roberto Muñoz é beneficiário da bolsa de Doutorado INF-PUCV 2015.

\section{Referências}

AEDO, C.; WALKER, I. Skills for the 21st Century in Latin America and the Caribbean. Washington, DC: The World Bank, 2012.

BARCELOS, T. S.; SILVEIRA, I. F. Pensamento Computacional e Educação Matemática: relações para o ensino de Computação na educação básica. XX Workshop sobre Educação em Computação. Anais do XXXII Congresso da Sociedade Brasileira de Computação. Curitiba: SBC, 2012.

BELL, T. et al. Computer Science Unplugged - An enrichment and extension programme for primary-aged children. Disponível em: <http://www.csunplugged.org>. Acesso em: 29 ago. 2015.

BOYCE, A. K. et al. Experimental evaluation of BeadLoom game: how adding game elements to an educational tool improves motivation and learning. ITiCSE ' 11. Proceedings of ITiCSE 2011. New York: ACM, 2011. p. 243-247. 
CBIE-LACLO 2015

Anais dos Workshops do IV Congresso Brasileiro de Informática na Educação (CBIE 2015)

CRESWELL, J. W.; MILLER, D. L. Determining validity in qualitative inquiry. Theory Into Practice, v. 39, n. 3, p. 124-130, 2000.

GROVER, S.; PEA, R. Computational Thinking in K-12: A Review of the State of the Field. Educational Researcher, v. 42, n. 1, p. 38-43, 2013.

HEMMENDINGER, D. A plea for modesty. ACM Inroads, v. 1, n. 2, p. 4-7, jun. 2010 .

HOOD, C. S.; HOOD, D. J. Toward integrating computing concepts into the K-12 curriculum. SIGCSE Bulletin, v. 37, n. 3, p. 375-375, jun. 2005.

HU, C. Computational thinking: what it might mean and what we might do about it. In: ITiCSE '11. Proceedings of ITiCSE 2011. New York: ACM, 2011. p. 223-227.

JENKINS, J. T.; JERKINS, J. A.; STENGER, C. L. A plan for immediate immersion of computational thinking into the high school math classroom through a partnership with the Alabama math, science, and technology initiative. ACM-SE '12. Proceedings of the 50th Annual Southeast Regional Conference. New York: ACM, 2012. p. 148-152.

KE, F. An implementation of design-based learning through creating educational computer games: A case study on mathematics learning during design and computing. Computers \& Education, v. 73, p. 26-39, 2014.

KRONE, J.; SITARAMAN, M.; HALLSTROM, J. O. Mathematics throughout the CS curriculum. Journal of Computing Sciences in Colleges, v. 27, n. 1, p. 65-73. 2011.

LEE, I. et al. Computational thinking for youth in practice. ACM Inroads, v. 2, n. 1, p. 32-37, 2011.

MAXWELL, A. et al. Robot RAL-ly International-Promoting STEM in elementary school across international boundaries using remote access technology. REV 2013. Proceedings of 2013 10th International Conference on Remote Engineering and Virtual Instrumentation. Sydney: IEEE, 2013. p. 1-5.

POLYA, G. How to solve it: a new aspect of mathematical method. Princeton: Princeton University Press, 2004.

TAUB, R.; ARMONI, M.; BEN-ARI, M. CS Unplugged and Middle-School Students' Views, Attitudes, and Intentions Regarding CS. Trans. Comput. Educ., v. 12, n. 2, p. $8: 1-8: 29,2012$.

THE CSTA STANDARDS TASK FORCE. CSTA K-12 Computer Science Standards. New York: ACM Computer Science Teachers Association, 2011. Disponível em: <http://csta.acm.org/Curriculum/sub/CurrFiles/CSTA_K-12_CSS.pdf>. Acesso em: 29 ago. 2015.

TROCHIM, W. M. K.; DONELLY, J. P. Research methods knowledge base. Disponível em: <http://www.socialresearchmethods.net/kb>. Acesso em: 29 ago. 2015, 2006

WING, J. M. Computational thinking. Communications of the ACM, v. 49, n. 3, p. 33-35, mar. 2006.

WOHLIN, C. et al. Systematic Literature Reviews. Experimentation in Software Engineering. Springer Berlin Heidelberg, 2012. p. 45-54. 